\title{
28 Research Square \\ The Axis-patella Index: A New Method for Measuring Patellar Height
}

\section{Hua Han ( $\nabla$ Izuhuahan@sina.com )}

Lanzhou University Second Hospital

Xiaohui Zhang

Lanzhou University Second Hospital

Research article

Keywords: patellar height, measurement, lateral radiograph, tibial shaft axis

Posted Date: November 5th, 2020

DOl: https://doi.org/10.21203/rs.3.rs-101436/v1

License: (9) This work is licensed under a Creative Commons Attribution 4.0 International License. Read Full License 


\section{Abstract}

Background: The widely used methods for measuring patellar height are based on the anatomic landmarks of the patella and the proximal tibia. When the knee undergoes alterations in the articular surface or adjacent structures due to various diseases or operations, the accurate identification of tibial landmarks is challenging. The results of these conventional methods may be biased or even unavailable. The purpose of this study was to introduce and validate a new method, axis-patella index (API), for assessing patellar height.

Methods: Patellar heights were respectively measured using the Insall-Salvati, modified Insall-Salvati, Blackburne-Peel, Caton-Deschamps, plateau-patellar angle, and axis-patella methods on the lateral radiographs of 649 knees without anatomic abnormalities. The inter- and intra-observer reliability of all methods were evaluated by calculating intraclass correlation coefficient and Bland-Altman plots. The associations between the API and the established methods were assessed using Pearson's correlation coefficient.

Results: The API was strongly correlated with the five aforementioned methods. Reliability analyses indicated high inter-observer reproducibility and intra-observer repeatability. The mean of API was approximately 1.5 , hence the reference range was defined $1.2-1.9$ according to the $95 \%$ confidence interval.

Conclusions: The API, a new index of referencing the tibial shaft axis and the fibular head, providing a valid and accurate alternative for the assessment of patellar height. The reference range was 1.2 - 1.9 in radiographically normal knee.

\section{Background}

Patellar height disorders can be highly correlated with various pathological conditions of the knee. Patella alta may cause patellofemoral pain and instability(1-3). Patella baja or infera may be congenital or commonly secondary to a trauma or iatrogenic factors, leading to the limitation of knee flexion and patellofemoral arthritis $(2,4,5)$.

Various measurement techniques devised for patellar height and the validation studies tested have increased in quantity and complexity. Currently, the most widely accepted radiographic techniques include Insall-Salvati index(ISI)(6), modified Insall-Salvati index (mISI)(7), Blackburne-Peel index (BPI) (8), Caton-Deschamps index(CDI)(9), and plateau-patellar angle (PPA)(2), as shown in Fig. 1. These techniques spatially relate the position of the patella to a marginal landmark of the proximal tibia(10).

Knee surgery or diseases involving the tibial plateau or tibial tubercle could increase the variability in the measurement of patellar height, and the results may be unreliable due to the loss of the tibial landmark. For instance, in patients undergoing knee arthroplasty, the tibial landmarks are often changed because of an alteration of the tibio-femoral joint line or posterior tibial slope $(5,11,12)$. In addition, in patients with 
severe osteoarthritis or rheumatoid arthritis, the identification of tibial landmarks using conventional methods are susceptible to osteophytes or articular surface destruction $(13,14)$, thereby causing a deviation of measurement.

Here, we conducted a new method for assessing patellar height, which spatially associates the articular surface of the patella with the tibial shaft axis, and theoretically can decrease the measurement deviation caused by identification difficulties of the tibial landmarks. The main objective of this study was to introduce and validate this new method for assessing patellar height. We proposed a hypothesis that this new method is reliably reproduced in radiographically normal knees, and it strongly correlated with classical methods.

\section{Methods}

\section{Patient selection}

After approval by the ethics committee in our institution, a retrospective analysis was performed on 2079 consecutive lateral radiographs of the knee (corresponding to 1521 patients) without preselection between Jan 2018 and June 2019. Radiographs had been made of all knees for the evaluation of clinical problems in both the outpatient department and casualty-department settings. The specific reasons for taking the radiographs were not clear, but it was assumed that there were different disorders. The exclusion criteria were as follows: (1) invisible or obscured bone landmarks on radiographs due to technical or pathological factors; (2) lateral radiographs showing in $<30^{\circ}$ of flexion; (3) radiographs of the knee of patients who presented with bone changes caused by fractures, arthroplasties, osteotomies, and other operations; (4) radiographs showing knee osteoarthritis with Kellgren-Lawrence grades $₫ 1$.

Of the total of 2079 radiographs, one hundred and twenty-seven (6.1\%) showed an obscure articular surface of the patella, tibial tubercle, and fibular head due to excessive knee rotation; sixty-nine (3.3\% ) were excluded because of the knee in $₫ 30^{\circ}$ of flexion; excluding four hundred and seventy-six $(22.9 \%)$ from the knees had undergone fractures, arthroplasties, osteotomies or other operations; seven hundred and fifty-eight (36.5\%) were excluded because of obvious tibio-femoral and/or patella-femoral osteoarthritis

with joint space narrowing and osteophytes formation. Eventually, 649 radiographs of 441 patients were measured actually.

\section{Measurement procedures}

The measurements of patellar height were taken on the lateral knee radiographs with the ST-PACS CDMedical software version 1.5.3 (Crealife, Beijing, China). Firstly, two trained observers performed a pilot study on 40 radiographs to validate the methodological feasibility and reach a technically detailed consensus. Two different observers (the authors) were involved in performing the measurements: observer 1 as a senior orthopedic surgeons and observer 2 as an orthopedic surgeons. Observer 1 independently measured all included radiographs. To assess the inter-observer reproducibility, observer 2 
independently measured 211 cases randomly selected from the total of 649 radiographs. Two observers were blinded about measurement results each other. To evaluate the intra-observer repeatability, 209 radiographs randomly selected from the original data set were measured by observer 1 with blinded manner after an interval of three months.

In this study, a new method named the axis-patella index (API) was proposed for assessing patellar height. This measurement method involves a length ratio $(a / b)$ between two line segments (Fig. 2$)$. The "a" is the distance from the lower pole of the patellar articular surface to the intersection point between the tibial shaft axis and its perpendicular line passing through the tip of the fibular head. The tibial shaft axis is the mid-diaphyseal line, as described by Shao et al(15). The "b" is the length of the articular surface of the patella.

Five conventional methods for assessing patellar height were conducted as described by the original authors in their published literature(2, 6-9). The ISI is the ratio between the length of the patellar tendon and the diagonal length of the patella (Fig. 1A). The length of the patellar tendon was measured from the lower pole of the patella to the insertion site of tendon on tibia. The patellar length was measured between the upper and lower pole of patella. The mISI is based on the length of the patellar articular surface and the distance from the tibial insertion of the patellar tendon to the inferior pole of the patellar articular surface (Fig. 1B). The length of the patellar articular surface was measured between the upper and lower pole of the articular surface of the patella. The BPI is calculated by dividing the perpendicular distance from the lower pole of the patellar articular surface to the anterior extension line of the medial tibial plateau by the length of the patellar articular surface (Fig. 1C). The CDI is the ratio of the distance between the lower pole of the articular surface and the antero-superior edge of tibia to the length of the articular surface of the patella (Fig. 1D). The PPA is measured between the tangent of the medial tibial plateau and the line connected from the lower pole of the articular surface of the patella to the posterior edge of the tibial plateau (Fig. 1E).

\section{Statistical analysis}

The data were analyzed with SPSS (version 25.0, SPSS Inc., Chicago, IL, USA). The results were expressed as arithmetic mean \pm standard deviation $(M \pm S d)$, and the normal distribution of API was tested. Descriptive statistics were performed to describe demographics and baseline data. The intraclass correlation coefficient (ICC) was used to analyze the inter-observer reproducibility and intra-observer repeatability.(16) Furthermore, the Medcalc software (version 11.4, MedCalc software Ltd., Acacialaan 22, 8400 Ostend, Belgium) was applied to construct the Bland-Altman plots showing the inter-observer and intra-observer variabilities in a visual way(16). The Pearson's correlation coefficient was calculated to determine the correlation between the API with other methods. $P<0.05$ was considered statistically significant. Lastly, we set the $95 \%$ confidence interval $(\mathrm{Cl})$ to establish the "reference range" $(\mathrm{M} \pm 1.96 * \mathrm{Sd})$ of API. 


\section{Results}

The demographics and the baseline data are shown in Table 1. The total of 649 radiographs were enrolled, $369(56.9 \%)$ in female and $280(43.1 \%)$ in male. The average age was $37.19 \pm 12.43$ years (range, 18-91), and the corresponding values were $38.14 \pm 12.41$ years in female (range, 18-79) and $35.93 \pm 12.35$ years in male (range, 18-91), respectively. The left knees were involved in $327(50.4 \%)$ cases and the right knees in 322 (49.6\%). The mean of API was $1.51 \pm 0.18$ (range, 1.05-2.10), and no significant differences were found between men (1.52 \pm 0.18 , range in 1.05-2.03) and women (1.49 \pm 0.18 , range in 1.05-2.10), $P=0.064$. The mean values of ISI, $\mathrm{mISI}, \mathrm{BPI}, \mathrm{CDI}$ and PPA were $1.02 \pm 0.12$, $1.69 \pm 0.19,0.90 \pm 0.14,0.98 \pm 0.15$, and $28.30 \pm 3.61$, respectively.

Table 1

Demographics and the baseline data.

\begin{tabular}{|lll|}
\hline Parameters & Mean \pm standard deviation & Range \\
\hline Sex (male : female) & $280: 369(43.1 \%: 56.9 \%)$ & \\
\hline Age (years) & $37.19 \pm 12.43$ & $18-91$ \\
\hline Side (left : right) & $327: 322(50.4 \%: 49.6 \%)$ & \\
\hline API & $1.51 \pm 0.18$ & $1.05-2.10$ \\
Male & $1.52 \pm 0.18$ & $1.05-2.03$ \\
Female & $1.49 \pm 0.18$ & $1.05-2.10$ \\
ISI & $1.02 \pm 0.12$ & $0.60-1.40$ \\
mISI & $1.69 \pm 0.19$ & $0.78-2.38$ \\
BPI & $0.90 \pm 0.14$ & $0.52-1.51$ \\
CDI & $0.98 \pm 0.15$ & $0.58-1.57$ \\
\hline PPA & $28.30 \pm 3.61$ & $17.80-39.96$ \\
\hline API = axis-patella index, ISI = Insall-Salvati index, m ISI = modified Insall-Salvati index, BPI = \\
Blackburne-Peel index, and CDI = Caton-Deschamps index, PPA = plateau-patella angle. \\
\hline
\end{tabular}

The measurement values of API were plotted as a frequency distribution along with a bell-shaped normal distribution curve with matching mean and standard deviation (Fig. 3). The distribution of this index could be identified by a normal distribution (continuous curve in Fig. 3). Consequently, we defined the reference range was 1.2-1.9 according the $95 \%$ confidence interval. The midpoint of a curve-fit to these data of a normal distribution was 1.5 , close to the mean of API.

Table 2 showed intraclass correlation coefficients, with $95 \% \mathrm{Cl}$, for inter-observer reproducibility and intraobserver repeatability. Inter-observer agreement in the API according to the ICC was 0.87 ( $95 \% \mathrm{Cl}$ : $0.83-$ 0.90 ) with the sample size of 211 , indicating excellent inter-observer reproducibility. The ICC between observers was 0.84 (95\% Cl: 0.80-0.88) for ISI, 0.73 (95\% Cl: 0.65-0.79) for mISI, 0.84 (95\% Cl: $0.79-$ 
0.87 ) for BPI, 0.83 (95\% Cl: $0.78-0.88)$ for $\mathrm{CDI}$, and 0.82 (95\% Cl: $0.77-0.86)$ for PPA, respectively. There were, therefore, small improvements for agreement using the API compared with other methods. In addition, the Bland-Altman plots illustrated the agreement among the investigated methods (Fig. 4), showing the mean difference (solid line) and $95 \%$ limits of agreement (dotted lines). The Bland-Altman plots depicted revealed, for the API, not only high inter-observer agreement but also no evidence of systematic biases, with a mean difference of 0.01 (95\% Cl: $0.00-0.03)$ being close to zero in a visual way.

Table 2

ICC of inter- and intra-observer concordance

\begin{tabular}{|c|c|c|c|c|c|c|c|c|}
\hline \multirow[t]{2}{*}{ Methods } & \multicolumn{4}{|c|}{ Inter-observer } & \multicolumn{4}{|c|}{ Intra-observer } \\
\hline & ICC & $95 \% \mathrm{Cl}$ & $\begin{array}{l}P \\
\text { value }\end{array}$ & $\begin{array}{l}\text { Sample } \\
\text { size }\end{array}$ & ICC & $95 \% \mathrm{Cl}$ & $\begin{array}{l}P \\
\text { value }\end{array}$ & $\begin{array}{l}\text { Sample } \\
\text { size }\end{array}$ \\
\hline API & 0.87 & $\begin{array}{l}0.83- \\
0.90\end{array}$ & 0.000 & 211 & 0.91 & $\begin{array}{l}0.89- \\
0.93\end{array}$ & 0.000 & 209 \\
\hline ISI & 0.84 & $\begin{array}{l}0.80- \\
0.88\end{array}$ & 0.000 & 211 & 0.88 & $\begin{array}{l}0.82- \\
0.92\end{array}$ & 0.000 & 209 \\
\hline mISI & 0.73 & $\begin{array}{l}0.65- \\
0.79\end{array}$ & 0.000 & 211 & 0.82 & $\begin{array}{l}0.77- \\
0.86\end{array}$ & 0.000 & 209 \\
\hline BPI & 0.84 & $\begin{array}{l}0.79- \\
0.87\end{array}$ & 0.000 & 211 & 0.83 & $\begin{array}{l}0.78- \\
0.87\end{array}$ & 0.000 & 209 \\
\hline CDI & 0.83 & $\begin{array}{l}0.78- \\
0.88\end{array}$ & 0.000 & 211 & 0.90 & $\begin{array}{l}0.87- \\
0.92\end{array}$ & 0.000 & 209 \\
\hline PPA & 0.82 & $\begin{array}{l}0.77- \\
0.86\end{array}$ & 0.000 & 211 & 0.88 & $\begin{array}{l}0.84- \\
0.91\end{array}$ & 0.000 & 209 \\
\hline
\end{tabular}

For intra-observer agreement of observer 1, the ICC in API was 0.91 (95\% Cl, 0.89-0.93), indicating high intra-observer repeatability. Moreover, good intra-observer agreements were also demonstrated in the ISI, $\mathrm{mISI}, \mathrm{CDI}, \mathrm{BPI}$ and PPA. More detailed results are presented in Table 2. These results were confirmed via the Bland-Altman analysis (Fig. 5), in which the differences of API scattered around zero with no systematic biases.

Table 3 demonstrated that all methods were strongly correlated when examining the five pairwise comparisons in the analysis of Pearson's correlation coefficient $(P<0.01)$. In particular, the API was more strongly correlated with BPI $(r=0.75, P<0.001), \mathrm{CDI}(r=0.73, P<0.001), \mathrm{mISI}(r=0.72, P<0.001)$, and PPA ( $r$ $=0.29, P<0.001)$. There was less degrees of correlation with the ISI but the correlation was still significant at $P=0.001(\mathrm{r}=0.14)$. 
Table 3

Correlations of API with ISI, mISI, BPI, CDI, and PPA

\begin{tabular}{|lllll|}
\hline & Comparison & Correlation coefficients & $\begin{array}{l}95 \% \text { confidence } \\
\text { interval }\end{array}$ & $P$ value \\
\hline API & ISI & 0.14 & $0.05-0.23$ & 0.001 \\
\hline API & $\mathrm{mISI}$ & 0.72 & $0.68-0.76$ & 0.000 \\
\hline API & BPI & 0.75 & $0.71-0.78$ & 0.000 \\
\hline API & CDI & 0.73 & $0.67-0.78$ & 0.000 \\
\hline API & PPA & 0.29 & $0.21-0.36$ & 0.000 \\
\hline $\begin{array}{l}\text { API = axis-patella index, ISI }=\text { Insall-Salvati index, m ISI = modified Insall-Salvati index, BPI = } \\
\text { Blackburne-Peel index, and CDI = Caton-Deschamps index, PPA = plateau-patella angle. }\end{array}$ \\
\hline
\end{tabular}

\section{Discussion}

The main contribution of this study is that the API was proposed for assessing patellar height. The evidences suggested the intraclass correlation coefficients of this new method were excellent, comparable with those of previously reported for ISI, $\mathrm{mISI}, \mathrm{BPI}, \mathrm{CDI}$ and PPA $(2,5,17)$. The API seems to have slightly better intra-observer repeatability and inter-observer reproducibility in more than 200 radiographs. Furthermore, the analyses of Pearson's correlation coefficient demonstrated strong correlations between the API and the aforementioned ones. Overall, the API is a valid and reliable alternative for assessing patellar height in radiographically normal knees.

Currently, the five published methods mentioned in this article are the most popular. However, there are inherent methodological difficulties, which may lead to an increase in inter- and intra-observer variability $(11,17-19)$. The ISI remains the most popular one, however, it is imperative to standardize the tibial attachment of the patellar tendon in order to avoid a significant variation resulting from the obscure morphology and the pathological overgrowth of the tibial tubercle $(18,20)$. Besides, the variants of patellar shape can lead to a greatly different result, especially in a patella being characterized by a long, non-articulating inferior pole (Cyrano patella)(19-21). The mISI described by Grelsamer et al(7) was designed to overcome the above disadvantages. Nonetheless, it did not provide the cutoff value for patellar baja. Blackburne and Peel(8) devised a method which exchanged the easily misidentified the tibial attachment of the patellar tendon for the tibial plateau as a landmark. Regrettably, they did not make a precise description of the tibial plateau line(12), resulting in the measurement variability. Portner et al(2) proposed the PPA which was formulated by the patella and tibial plateau. However, it is commonly found that the posterior margin of tibial plateau was rounded and smooth, making it difficult to unify the vertex of the angle in repeatable measurements. Caton et al(9) described that the anterosuperior edge of the tibia was used as the landmark. Whereas an accurate identification of the reference 
could be difficult in osteoarthritic knees with osteophytes $(12,14)$. In view of this, it is necessary to find an accurate measurement method that adopts new reference to avoid the above identification difficulties.

The tibial landmark of the API, formed by the tibial shaft axis and its perpendicular line passing through the tip of the fibular head, intrinsically owns better positional stability. Compared with the tibial plateau, the fibular head is independent of osteophytes and articular surface destruction. And the recognition of the styloid process of fibular head is accurately acquired on the standard lateral radiograph. Meanwhile, the tibial shaft axis is an independent reference with spatial constancy. In this study, it was determined by selecting two mid-diaphyseal points at 70 - and $110-\mathrm{mm}$ distal to the tibial plateau down the tibial shaft, in which could avoid an increase of the measurement variability, as described by previous studies $(15,22)$. As an intramedullary reference, the tibial shaft axis can theoretically provide a constant landmark compared to marginal counterparts, especially during tibial rotating subsequent to knee flexion. Thus, we speculated that maintaining the greater accuracy and less variability in the measurements could be based on the above procedures.

Consistent with standards of previous methods, this new method is independent of radiographic magnification, physical size of the knee, and angles of knee flexion $(2,7,8,12)$. In this study, as mentioned above, the radiologically normal knees were enrolled in an endeavour to establish a normal range of values. The results demonstrated that the average value of API is approximately 1.5 using the sample size of 649 radiographs, thereby the reference range is recommended as $1.2-1.9$ based on the $95 \%$ confidence interval. When the value is greater than 1.9 or less than 1.2 , surgeons should be alert to the occurrence of patella alta or infera. We also evaluated the correlations between the API and other five methods, and delighted to find that the new method was strongly correlated with the mISI, BPI and CDI. This is partially explained by the fact that these four methods share the patellar articular surface. Moreover, the results showed that the API was less correlated with ISI and PPA, and a potential explanation might be the influence of the anatomic variability of patellar shape, as illustrated by Grelsamer et al(20).

There are several limitations of the present study that warrant consideration. Firstly, this study employed a retrospective design and the case data were extracted from the hospital information system. The subjects were rated as radiologically normal, but the clinical symptoms could not be determined and injuries of meniscuses or cruciate ligaments might be included. However, these soft-tissue problems could be deemed acceptable on the assumption that the patellofemoral relationship is little affected, as mentioned in the previous studies $(6,8,9)$. Secondly, variabilities in the distance between the tip of the fibular head and the joint line should not be ignored, and the reference range derived from this study ought to be treated with caution. Thirdly, the authors speculated that the API cannot be used when the fibular head is damaged by bone neoplasms, or in patients who have had the fibular head removed. Finally, the samples included in this study were radiographically normal knees, it is necessary to investigate the application of the API in pathological conditions in further studies. Despite the limitations set out above, there are still several design advantages, including a large sample size, measuring patellar 
height with six different methods, and simultaneously assessing the inter- and intra-observer reliability in all methods.

\section{Conclusions}

In conclusion, the API, a new index of referencing the tibial shaft axis and the fibular head, providing a valid and accurate alternative for the assessment of patellar height. The reference range was 1.2-1.9 in radiographically normal knee.

\section{List Of Abbreviations}

ISI, Insall-Salvati index; mISI, modified Insall-Salvati index; BPI, Blackburne-Peel index; CDI, CatonDeschamps index; PPA, plateau-patellar angle; API, axis-patella index; ICC, intraclass correlation coefficient; $\mathrm{Cl}$, confidence interval.

\section{Declarations}

\section{Ethics approval and consent to participate}

Ethics approval for this study was obtained from the Institutional Review Board of the Lanzhou University Second Hospital, Lanzhou, Gansu Province, China. The informed consent was waived by the Ethics Committee as the study was performed using the data and images without the patients identification information and this is a retrospective study.

\section{Consent for publication}

Not applicable.

\section{Availability of data and material}

The data and materials in current paper may be made available upon request through sending e-mail to first author.

\section{Competing interests}

The authors declare that they have no competing interests.

\section{Funding}


This work was supported by the Gansu provincial Construction project of traditional Chinese medicine inheritance and innovation platform [TCM-IPC-2020-01].

\section{Authors' contributions}

$\mathrm{HH}$ designed the study; $\mathrm{HH}$ and $\mathrm{XHZ}$ collected, analyzed and interpreted the data; $\mathrm{HH}$ and $\mathrm{XHZ}$ wrote the initial draft, ensured the accuracy of the data. All authors read and approved the final manuscript.

\section{Acknowledgements}

Not applicable.

\section{References}

1. Mayer C, Magnussen RA, Servien E, Demey G, Jacobi M, Neyret P, et al. Patellar tendon tenodesis in association with tibial tubercle distalization for the treatment of episodic patellar dislocation with patella alta. Am J Sports Med. 2012;40(2):346-51.

2. Portner O, Pakzad H. The evaluation of patellar height: a simple method. J Bone Joint Surg Am. 2011;93(1):73-80.

3. Lewallen LW, Mclntosh AL, Dahm DL. Predictors of recurrent instability after acute patellofemoral dislocation in pediatric and adolescent patients. Am J Sports Med. 2013;41(3):575-81.

4. Aparicio G, Abril JC, Calvo E, Alvarez L. Radiologic study of patellar height in Osgood-Schlatter disease. Journal of pediatric orthopedics. 1997;17(1):63-6.

5. Rogers BA, Thornton-Bott P, Cannon SR, Briggs TW. Interobserver variation in the measurement of patellar height after total knee arthroplasty. The Journal of bone and joint surgery British volume. 2006;88(4):484-8.

6. Insall J, Salvati E. Patella position in the normal knee joint. Radiology. 1971;101(1):101-4.

7. Grelsamer RP, Meadows S. The modified Insall-Salvati ratio for assessment of patellar height. Clin Orthop Relat Res. 1992(282):170-6.

8. Blackburne JS, Peel TE. A new method of measuring patellar height. The Journal of bone and joint surgery British volume. 1977;59(2):241-2.

9. Caton J. Method of measuring the height of the patella. Acta orthopaedica Belgica. 1989;55(3):3856.

10. Biedert RM, Tscholl PM. Patella Alta: A Comprehensive Review of Current Knowledge. American journal of orthopedics (Belle Mead, NJ). 2017;46(6):290-300.

11. Caton JH, Prudhon JL, Aslanian T, Verdier R. Patellar height assessment in total knee arthroplasty: a new method. Int Orthop. 2016;40(12):2527-31. 
12. Phillips CL, Silver DA, Schranz PJ, Mandalia V. The measurement of patellar height: a review of the methods of imaging. The Journal of bone and joint surgery British volume. 2010;92(8):1045-53.

13. Cabral F, Sousa-Pinto B, Pinto R, Torres J. Patellar Height After Total Knee Arthroplasty: Comparison of 3 Methods. The Journal of arthroplasty. 2017;32(2):552-7.e2.

14. Berg EE, Mason SL, Lucas MJ. Patellar height ratios. A comparison of four measurement methods. Am J Sports Med. 1996;24(2):218-21.

15. Shao H, Chen C, Scholl D, Faizan A, Chen AF. Tibial shaft anatomy differs between Caucasians and East Asian individuals. Knee surgery, sports traumatology, arthroscopy : official journal of the ESSKA. 2018;26(9):2758-65.

16. Langlois J, Hamadouche M. Current recommendations for assessing the reliability of a measurement tool: a survival guide for orthopaedic surgeons. The bone \& joint journal. 2016;98$\mathrm{b}(2): 166-72$.

17. van Duijvenbode D, Stavenuiter M, Burger B, van Dijke C, Spermon J, Hoozemans M. The reliability of four widely used patellar height ratios. Int Orthop. 2016;40(3):493-7.

18. Seil R, Müller B, Georg T, Kohn D, Rupp S. Reliability and interobserver variability in radiological patellar height ratios. Knee surgery, sports traumatology, arthroscopy : official journal of the ESSKA. 2000;8(4):231-6.

19. Kadakia NR, llahi OA. Interobserver variability of the Insall-Salvati ratio. Orthopedics. 2003;26(3):3213; discussion 3-4.

20. Grelsamer RP, Proctor CS, Bazos AN. Evaluation of patellar shape in the sagittal plane. A clinical analysis. Am J Sports Med. 1994;22(1):61-6.

21. Grelsamer RP. Patella baja after total knee arthroplasty: is it really patella baja? The Journal of arthroplasty. 2002;17(1):66-9.

22. Tang Q, Zhou Y, Yang D, Xu H, Liu Q. The offset of the tibial shaft from the tibial plateau in Chinese people. J Bone Joint Surg Am. 2010;92(10):1981-7.

\section{Figures}




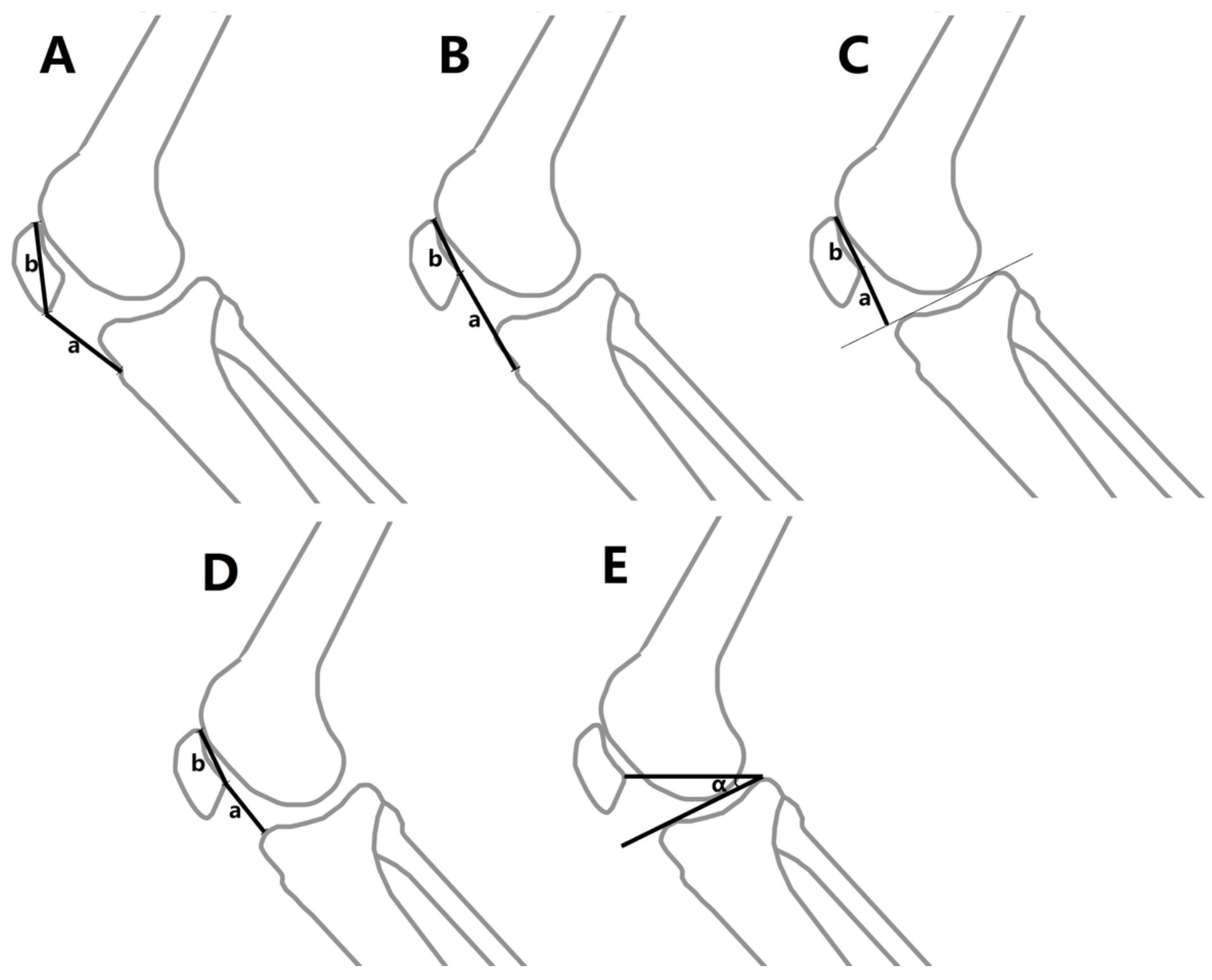

Figure 1

Representation of the five widely used methods for assessing patellar height. (A). Insall-Salvati index (ISI: a/b); (B). modified Insall-Salvati index (mISI: a/b); (C). Blackburne-Peel index (BPI: a/b); (D). Caton-Deschamps index (CDI: a/b); (E). plateau-patellar angle (PPA). 


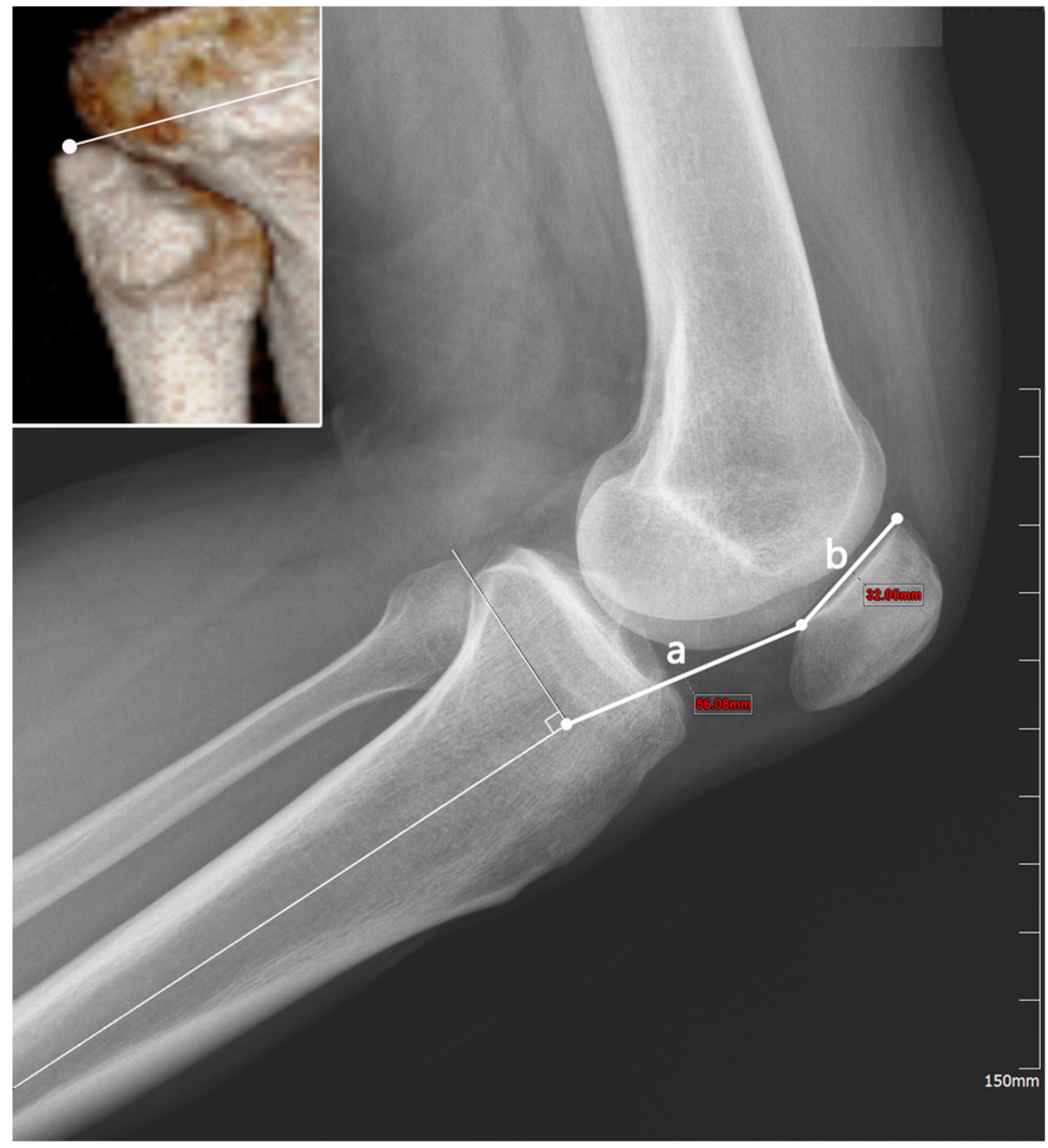

\section{Figure 2}

The axis-patella index measures the distance between the lower pole of the patellar articular surface to the intersection point between the tibial shaft axis and its perpendicular line passing through the tip of the fibular head (a) divided by the length of the articular surface of the patella (b). Partial highlighted view showing a tangent line passing through the styloid process of the three-dimensional reconstructed fibula head by computed tomography. 


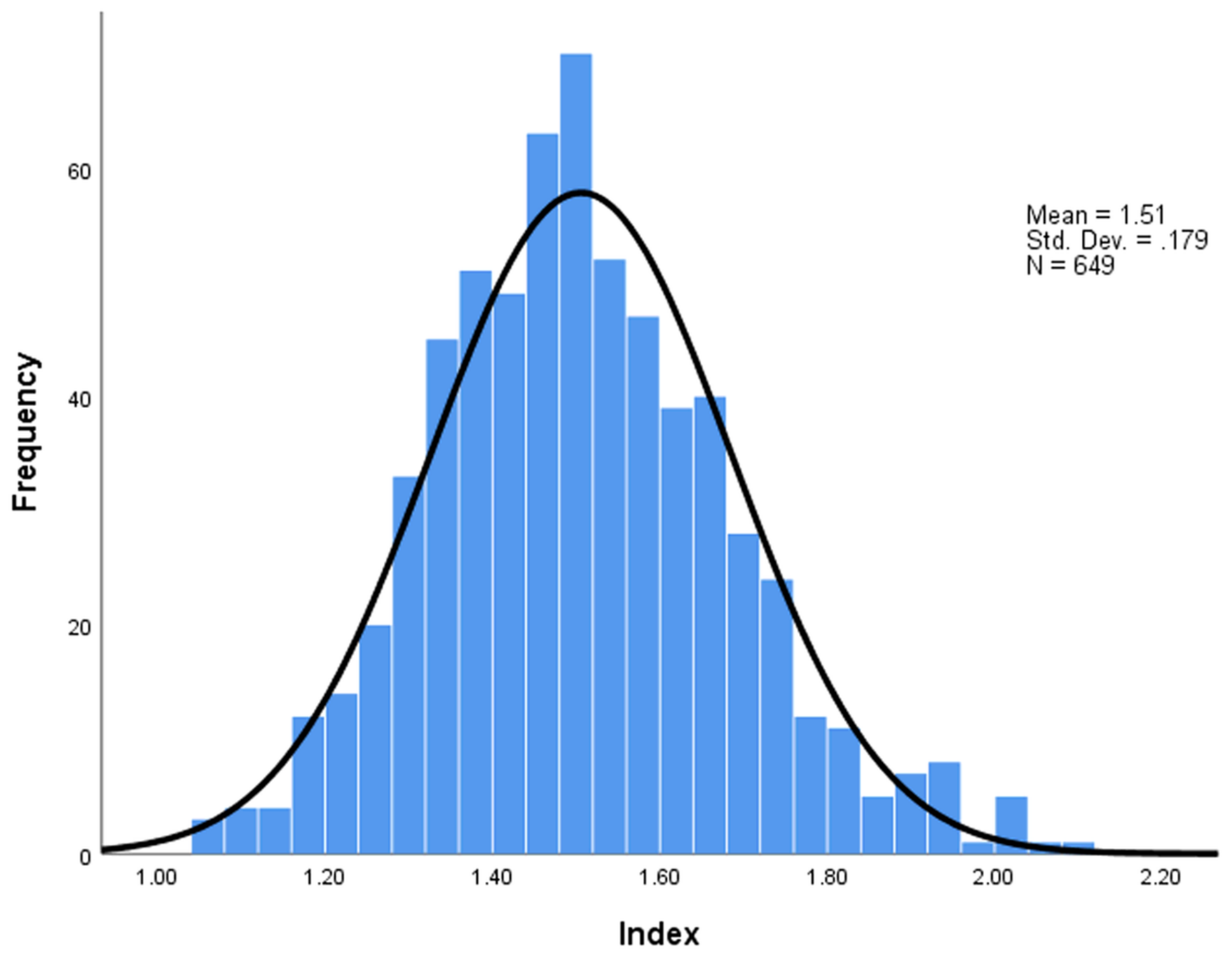

Figure 3

The histogram showing the bell-shaped normal distribution of the axis-patella index for measuring the height of the patella. Mean $=1.51$. Std. Dev $=$ standard deviation $=0.179$. 

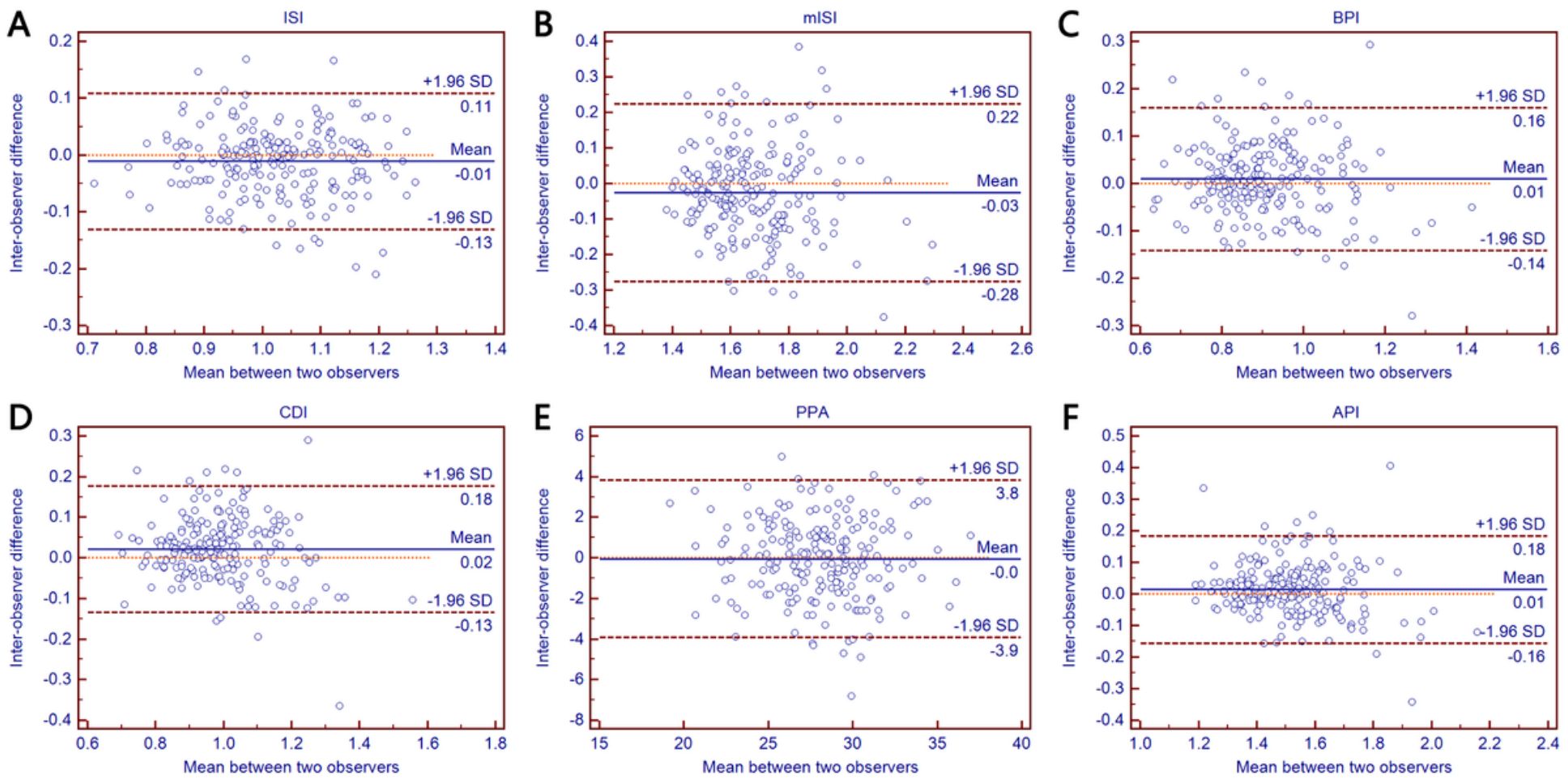

Figure 4

Bland-Altman plots of the differences for measuring patellar height between two observers: (A) InsallSalvati index (ISI); (B) modified Insall-Salvati index (mISI); (C) Blackburne-Peel index (BPI); (D) CatonDeschamps index (CDI); (E) plateau-patellar angle (PPA); (F) axis-patella index (API).
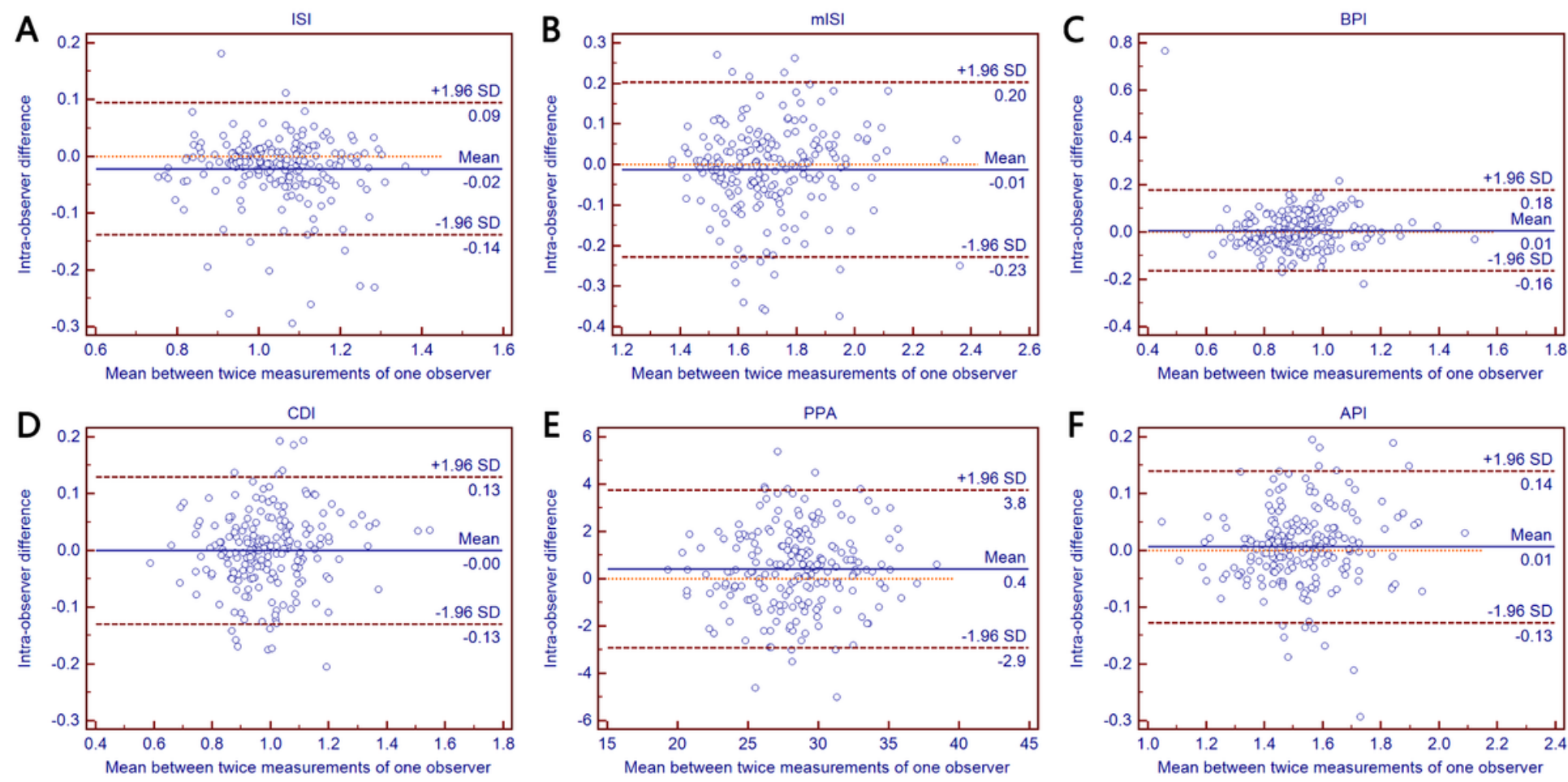

Figure 5 
Bland-Altman plots of the differences for measuring patellar height between twice measurements by one observer after a 3 months interval time: (A) Insall-Salvati index (ISI); (B) modified Insall-Salvati index (mISI); (C) Blackburne-Peel index (BPI); (D) Caton-Deschamps index (CDI); (E) plateau-patellar angle (PPA); (F) axis-patella index (API). 